\title{
Expression of Prostate-Specific Membrane Antigen (PSMA) in Brain Glioma and its Correlation with Tumor Grade
}

\author{
Hiva Saffar ${ }^{1 *}$, Maryam Noohi ${ }^{1}$, Seyed Mohammad Tavangar $^{1}$, Hana Saffar $^{2}$, Sima Azimi $^{1}$ \\ 1. Dept. of Pathology, Shariati Hospital, Tehran University of Medical Sciences, Iran \\ 2. Dept. of Pathology, Imam Hospital Complex, Tehran University of Medical Sciences, Iran
}

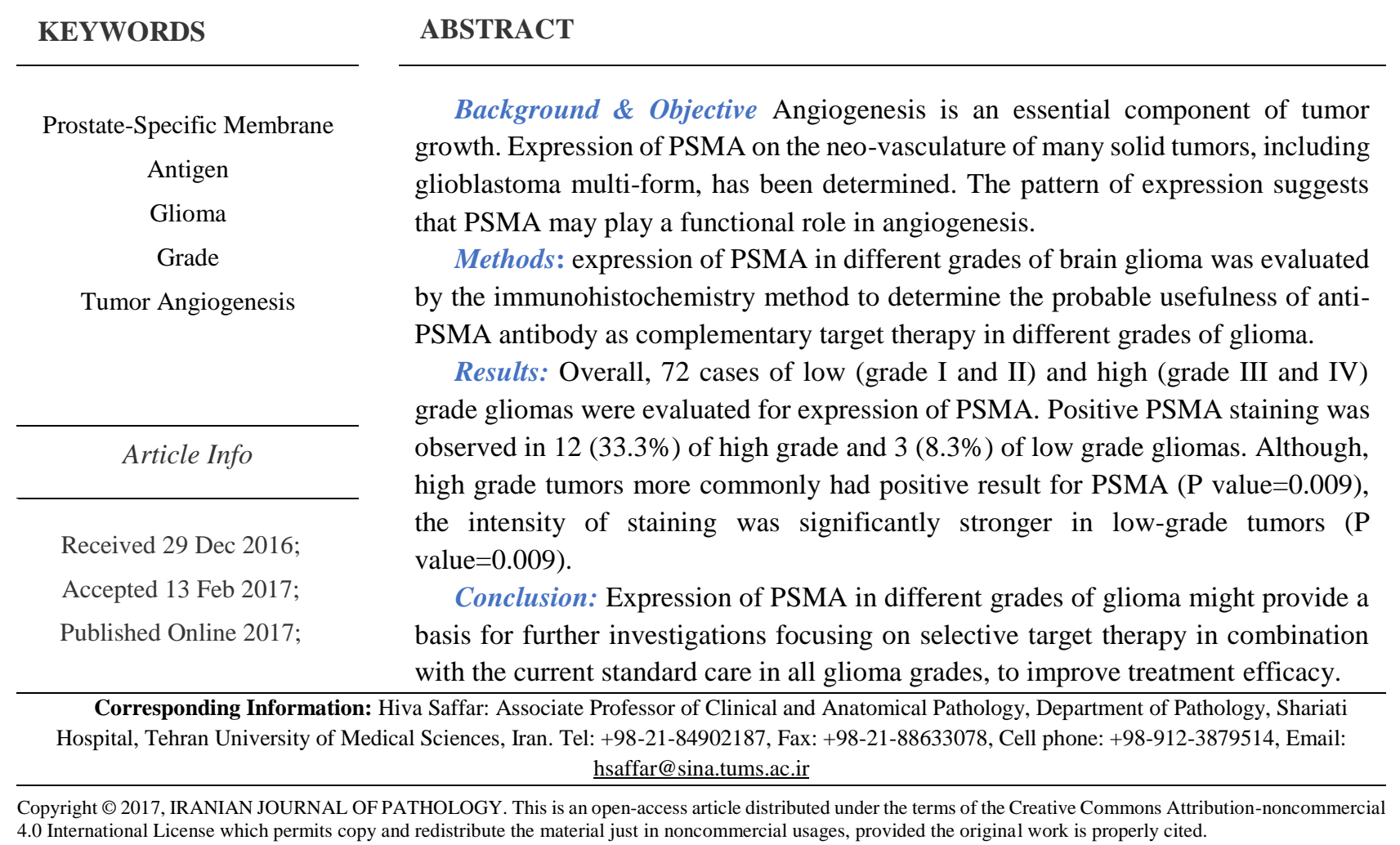

\section{Introduction}

Gliomas are the most prevalent primary brain tumors (1-3) with a median survival of about one year (3). While grades I, II, and III progress to a poor outcome over 2 to 10 years, grade IV behaves more aggressively (2) and despite treatment improvements 5-year survival of Grade IV glioma (Glioblastoma Multi-form:,GBM) still remains poor and reported less than 5\% (1, 3-5).

Angiogenesis is an essential component of tumor growth (6). The signals could be related to environmental or genetic alterations in the tumor (6). Vascular Endothelial Growth Factor (VEGF) is one of the stimulants of endothelial activation, believed to be secreted by almost all solid tumors, including glioma $(6,7)$. Hypoxia promotes VEGF (6), hich correlates with microvasculature

formation and tumor growth and has been targeted to treat high-grade glioma $(\mathrm{GBM})(8,9)$.

On the other hand, regarding abnormal phenotype of tumor vessels, there is great interest in understanding the underlying genetic alteration of tumor angiogenesis and differences in protein expression, which might provide potential tumorspecific targets for therapy (6, 10-12). Prostate Specific Membrane Antigen (PSMA) is a type II integral membrane glycoprotein, which was initially determined in prostate cancer cells, yet was later discovered to be robustly expressed in neovasculature of a variety of solid tumors, including $\operatorname{GBM}(2,13)$; this suggests the possible role of PSMA in tumor angiogenesis (14).

In brain tumors, because the tumor substance could be protected by the Blood Brain Barrier 
(BBB), the endothelium is commonly exposed to a potential radio-labeled antibody or antibody conjugated by cytotoxin. Therefore, targeting the endothelium in brain tumors appears more attractive (6).

In histology, GBM reveals high vessel density, indicating significant angiogenic activity (15). The presence of PSMA, as a potential promising target in some tumors, including GBM, has been previously evaluated $(2,6,16-18)$. However, data about lower grade gliomas is limited (2).

Since despite treatment lower grade gliomas often progress and transform to higher grades and more aggressive forms, using novel therapeutic methods and new strategies offers complementary treatment options for all grades of glioma.

In this study, the researchers evaluated the expression of PSMA in different grades of brain glioma by the immunohistochemistry method to determine the probable usefulness of Anti-PSMA antibody, as complementary target therapy in glioma. Also, the expression of PSMA was compared with VEGF, to determine possible alternative roles in tumor angiogenesis.

\section{Materials and Methods}

Paraffin blocks of formalin-fixed samples diagnosed as glioma (astrocytoma) during 2011 and 2014 were retrieved from the archives of the department of pathology, Shariati Hospital, Tehran, Iran. The slides were examined and reclassified according to the World Health Organization (WHO) classification. The questionable cases were excluded. The tumors were divided to high and low grades, including 10 grade I and 26 grade II samples, categorized as low grade, and 9 grade III and 27 grade IV, classified as high grade. Paraffin blocks with representative amounts of viable tumor tissue were selected and sectioned at $4 \mu \mathrm{m}$ intervals for the Immunohistochemistry study. Immunohistochemistry was performed using monoclonal liquidNovocastra ${ }^{\mathrm{TM}}$-Liquid- Mouse monoclonal antibody (clone 1D6, Novocastra) and Rabbit polyclonal VEGF Antibody (Pu483-up, BoiGenex), according to the manufacturers' instructions. For VEGF, certain modifications were considered. Briefly, sections were deparaffinized, rehydrated and incubated in buffer solution $\left(60^{\circ} \mathrm{C}\right.$,
$\mathrm{PH}=9$ ) for one hour. After 15 minutes at room temperature, samples were placed in $3 \%$ hydrogen peroxide for 60 minutes and washed with distilled water. Non-specific binding was blocked with a 30minute wash in free serum protein block solution (Dako). The anti-VEGF antibody in a 1:15 dilution was used and the slides were incubated at $4{ }^{\circ} \mathrm{C}$ overnight. The slides were then placed at room temperature for 15 minutes, and washed with TrisBuffered Saline (TBS) buffer solution. Next, secondary anti-body (1:15 dilution, super sensitive TM Polymer-HRP IHC Detection System, Bio Genex) was added and the samples were incubated for 30 minutes followed by washing with buffer solution.

Prostate tissue and capillary hemangioma were considered as control tissue for PSMA and VEGF, respectively (Figure 1).

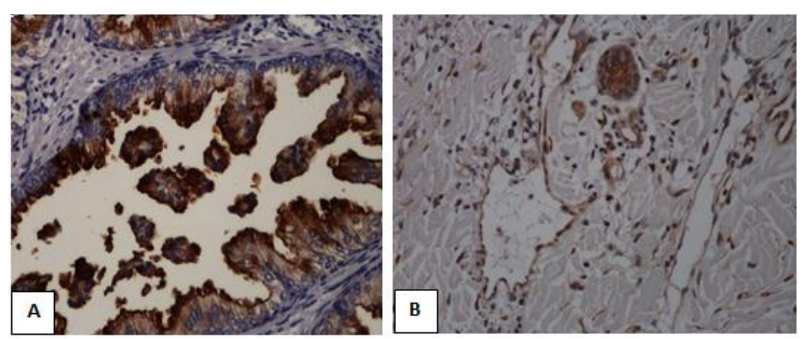

Fig 1. Prostate as a positive control for PSMA (A) and Hemangioma as a positive control for VEGF (B).

Also, a CD31 (monoclonal Antibody, JC/70A, BioGenex) study was performed to confirm localization of neo-vasculature endothelium.

The stained sections were assessed for the intensity and extent of endothelial cell staining in tumor micro-vessels and scored semi-quantitatively (Tables I and II) (Figures 2 and 3).

After data collection, the analysis of quantitative and qualitative data was performed using the Statistical Package for Social Science (SPSS) version 19 (IBM corporation). $\mathrm{P}$ values of $<0.05$ were considered statistically significant. 


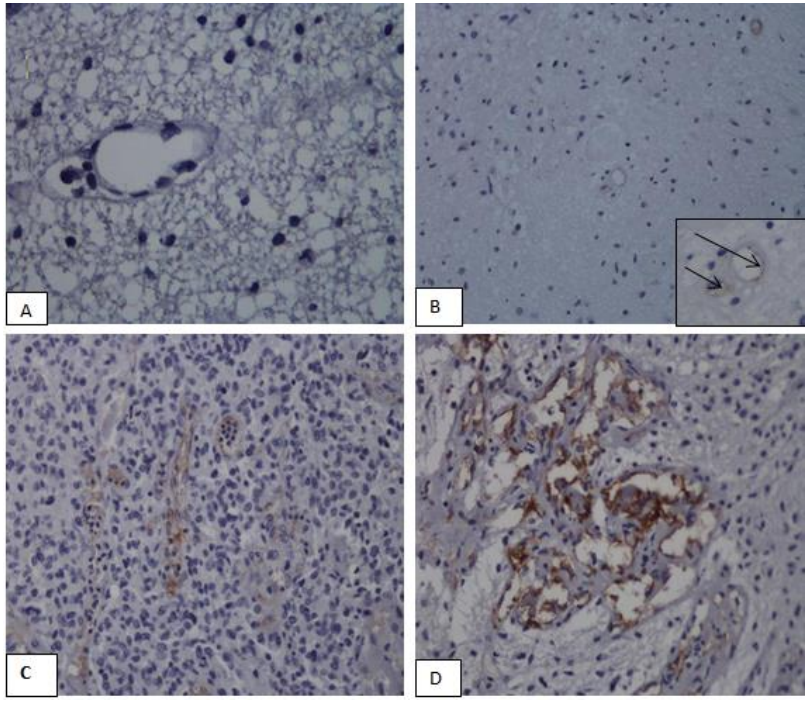

Fig 2. Different patterns of intensity of PSMA staining: Score 0 in diffuse glioma WHO grade II(A); Score 1 in diffuse glioma WHO grade II (B); Score 2 in diffuse glioma WHO grade III (C); Score 3 in diffuse glioma WHO grade IV (D)

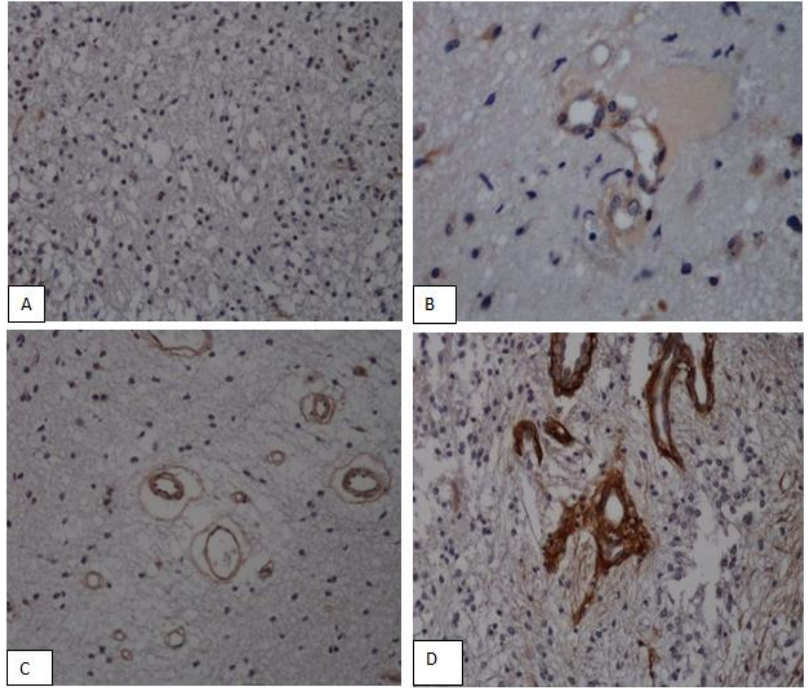

Fig 3. Different patterns of intensity of VEGF staining: Score 0 in diffuse glioma WHO grade II(A); Score 1 in diffuse glioma WHO grade II (B); Score 2 in diffuse glioma WHO grade II (C); Score 3 in diffuse glioma WHO grade IV(D)

Table 1. Interpretation of IHC Results Regarding "Extent" of Staining

\begin{tabular}{|cc|} 
Percentage of stained endothelial cells $(\%)$ & Interpretation \\
\hline $\mathbf{0}$ & Negative \\
\hline $10-9$ & Minimal \\
\hline $40-69$ & Moderate \\
\hline$>70$ & Strong \\
\hline
\end{tabular}

Table 2. Interpretation of IHC Results Regarding "Intensity" of Staining

\begin{tabular}{|cc}
\hline Intensity & Interpretation \\
\hline Faint and weak staining at High power & $1+$ \\
\hline Moderate intensity at low power & $2+$ \\
\hline Strong reaction at low power & $3+$ \\
\hline
\end{tabular}

\section{Results}

Positive result for PSMA staining was observed in $12(33.3 \%)$ of the high grade and $3(8.3 \%)$ of the low-grade gliomas, including 2, 1, 1 and 11 positive results of samples in grade I, II, III and IV, respectively. A statistically significant association was observed between PSMA expression and tumor grade $(\mathrm{P}$ value $=0.009)$, meaning that high grade gliomas more commonly had positive results for PSMA; although most of them had negative findings. 
Moreover, the researchers determined that the intensity of staining was significantly stronger in low-grade tumors $(\mathrm{P}$ value $=0.009)$ (Table 3).

The extent of PSMA expression was wider in high grade gliomas, yet this was not significant ( $\mathrm{P}$ value $=0.693)($ Table 4).
The VEGF had positive result in all tumoral vessels and high grade tumors showed stronger reaction in comparison with low grade gliomas $(\mathrm{P}$ value $=0.052)$ (Tables 5 and 6).

Table 3. The Intensity of PSMA Staining in Different Grades of Glioma

\begin{tabular}{cccccc}
$\begin{array}{c}\text { Interpretation } \\
\text { Of Grade }\end{array}$ & IV & $9 / 27$ & $\mathbf{2 +}$ & $\mathbf{3 +}$ & Total \\
\hline High grade & IV & $1 / 9$ & 0 & $1 / 27$ & $11 / 27$ \\
\hline Low grade & III & 0 & $1 / 26$ & 0 & $1 / 9$ \\
\hline & II & 0 & $2 / 10$ & 0 & $1 / 26$ \\
\hline -value $=0.009$ & I & & & 0 & $2 / 10$
\end{tabular}

Table 4. The Extent of Prostate-Specific Membrane Antigen (PSMA) Staining at Different Grades of Glioma

\begin{tabular}{cc|ccccccc}
$\begin{array}{c}\text { Extent of Staining } \\
\text { Grade }\end{array}$ & & $\mathbf{0}$ & $\mathbf{1 - 9} \%$ & $\mathbf{1 0 - 3 9 \%}$ & $\mathbf{4 0 - 6 9 \%}$ & $\mathbf{7 0 \%}$ & Total \\
\hline \multirow{2}{*}{ High-grade } & IV & $\mathbf{1 6 / 2 7}$ & $4 / 27$ & $5 / 27$ & $1 / 27$ & $1 / 27$ & $27 / 27$ \\
\cline { 2 - 8 } & III & $8 / 9$ & 0 & 0 & 0 & $1 / 9$ & $9 / 9$ \\
\hline Low-grade & II & 0 & 0 & 0 & 0 & $1 / 26$ & $26 / 9$ \\
\hline$P$-value $=0.693$ & I & $8 / 10$ & 0 & $2 / 10$ & 0 & 0 & $10 / 10$ \\
\hline
\end{tabular}

Table 5. The Intensity of Vascular Endothelial Growth Factor Staining in Low and High Grades of Glioma

\begin{tabular}{|c|c|c|c|c|}
\hline $\begin{array}{c}\text { Interpretation } \\
\text { Grade }\end{array}$ & $1+$ & $2+$ & $3+$ & Total \\
\hline High Grade & 0 & $12 / 36$ & $24 / 36$ & $36 / 36$ \\
\hline Low Grade & 0 & $5 / 36$ & $31 / 36$ & $36 / 36$ \\
\hline
\end{tabular}

Table 6. The Extent of Vascular Endothelial Growth Factor Staining in Low and High Grades of Glioma

\begin{tabular}{ccccccc}
$\begin{array}{c}\text { Interpretation } \\
\text { Grade }\end{array}$ & $\mathbf{0}$ & $\mathbf{1 - 9} \%$ & $\mathbf{1 0 - 3 9 \%}$ & $\mathbf{4 0 - 6 9 \%}$ & $\mathbf{> 7 0 \%}$ & Total \\
\hline High-grade & 0 & 0 & $2 / 36$ & $2 / 36$ & $32 / 36$ & $36 / 36$ \\
\hline Low-grade & 0 & 0 & 0 & $4 / 36$ & $32 / 36$ & $36 / 36$ \\
\hline$P$-value $=0.433$ & & & &
\end{tabular}

No significant correlation was observed between the extent and intensity of PSMA with VEGF $(0.634$ and 0.398 , respectively).

The mean age of patients was $46 \pm 14.4$ and $25.6 \pm 10.1$ years in high- and low-grade tumors, respectively.

In addition, patients with tumors showing a positive reaction to PSMA were older on average
(44.5 years old versus 33.7 years old) ( $\mathrm{P}$ value $=0.020$ ).

\section{Discussion}

The PSMA is a highly specific $(6)$ type II $(6,19)$ trans-membrane protein (14) located on chromosome $11 \mathrm{p}$ (20). This membrane was initially thought to be prostate specific (20), however, 
further studies discovered that cells of the small intestine, proximal renal tubules, and salivary glands express PSMA (19-21).

The function of PSMA is currently under investigation (6). It has been characterized that PSMA has folate hydrolase activity and some studies showed that it also has a neuropeptidase type function $(6,19,20)$.

Moreover, expression of PSMA on the neovasculature of many solid tumors, including GBM, has been shown by previous studies $(2,6,19,20)$. Although the significance has not been fully determined, the pattern of expression suggests that PSMA may play a functional role in angiogenesis (14). It is anchored to the cell membrane, which makes it an ideal promising therapeutic target (6, 14). In addition, it can be internalized making it an appropriate candidate for pro-drug activity $(6,14$, 22). In-vivo, anti-PSMA antibody has been used successfully against the neo-vasculature of a number of solid non-prostate tumors $(6,23,24)$.

The luminal side of intestinal epithelium is not accessible via vasculature and PSMA targeted antibodies are too large to filter to the glomeruli, thus the antibody cannot reach the apical surface of renal tubular epithelial cells. Hence, targeting PSMA on endothelial cells of tumor vasculature can selectively destroy tumor vessels and rather spare normal tissue, which lacks PSMA expression (2, 25).

In brain tumors, including GBM, BBB protects tumor mass, at least partially, so the endothelium of brain tumor can be exposed much more readily than tumor substance $(2,6)$. Also, probable low-level heterogeneous expression of PSMA in normal brain can be protected by BBB $(2,26)$.

In a study by Wernicke et al., (6) 32 cases of GBM were evaluated, all of them revealed PSMA expression in their neo-vasculature, regardless of the morphology of endothelial cells. They concluded that PSMA could be a potential novel therapeutic vascular target (6).

In another study by Mhawech-Fauceglia et al., $(6,18) 49$ of 52 GBM samples evaluated by IHC using microarray, had negative results for PSMA. Two samples had weakly and one moderately positive results. No strong reaction was observed. The researchers did not report any neo-vasculature expression due to minimal stroma in their samples. These were in contrast with the former study by Wernicke et al. (6), which explained that the discrepancy is probably due to using different clones of anti-PSMA antibodies (3E6 mAb by the former versus y-PSMA-1 by the latter).

In addition, none of the PSMA positive nonprostate tumors (including GBM) evaluated by Mhawech-Fauceglia et al. $(6,18)$ showed membrane staining despite the fact that PSMA is a trans-membrane protein (6). This also supports the explanation considered by Wernicke et al. (6).

A number of previous studies have evaluated PSMA expression in $\operatorname{GBM}(2,6,18,19)$, however, the data on lower grade gliomas is limited (2).

In the current study, the glioma cases were divided into high- and low-grade categories. Highgrade gliomas significantly more commonly had positive results for PSMA ( $\mathrm{P}$ value $=0.009$ ). In highgrade tumors (including grades III and IV), 12 of 36 $(33.33 \%)$ cases showed positive reaction to PSMA while only 3 of $36(8.33 \%)$ low-grade tumors had positive finding for PSMA.

Of 12 positive high-grade tumors, 1 and 11 cases were categorized as grade III and IV, respectively. Thus, not all cases of GBM had positive result for PSMA, which is in contrast with the observations of Wernicke et al. (6). In a study by Nomura et al. (2), formalin-fixed paraffin embedded samples of primary and secondary brain tumors were evaluated for PSMA expression. Samples of grade I (5), II (4), III (5) and IV(5) as well as 4 cases of normal brain were included in this study. The expression level was imaged and quantified using automated hardware and software. Their assessment revealed that GBM cases had strongly positive results for PSMA, and grade II and III tumors exhibited staining in tumor parenchyma with no endothelial cell staining. In grade I gliomas, the vessels showed moderate staining. The vessels of the normal brain had negative results for PSMA (2).

According to the current findings, 2 cases in grade I and 1 case in grade II and III had positive results for PSMA, respectively.

The overall positive reaction was significantly more in high-grade tumors, yet no significant correlation was determined between the extent of PSMA staining and tumor grade. However, the 
intensity of endothelial cell staining was stronger in low-grade tumors (the 3 positive low grade gliomas revealed $2+$ intensity) $(\mathrm{p}=0.009)$, which the former is in concordance with the findings reported by Nomura et al. (2).

As mentioned previously, different patterns of PSMA expression among several reported studies could be due to using various clones of antibody targeting different antigenic epitopes.

The current study used Novocastra liquid NCLL- PSMA Clone 1D6, while both the previous mentioned studies (6) (2) performed IHC staining by Anti-PSMA mAb 3E6 DAKO (Carpentaria, CA).

Chang et al., (19), evaluated 5 different Anti PSMA Antibodies (7E11, J591, J415, HybritechPEQ226.5 and PM2Joo4.5), each bounded a distinct epitope of PSMA. They concluded that, with rare exceptions, all five antibodies reacted with neo-vasculature of a vast majority of malignant neoplasms, including one case of GBM (19).

The clone used in the current research was not included in the latter study and only 1 case of GBM was included in the study (19). Therefore, the researchers suggest that regarding variable or contradictory results, the extent of PSMA staining within different glioma grades needs to be further investigated in large-scale studies.

The VEGF is another angiogenic factor released by many tumor cells (22). Hypoxia promotes VEGF secretion (6) and VEGF simulates angiogenesis resulting in increased vascularity (22).

Intact VEGF and its receptor (VEGFr3VEGFR2/KDR) are determined as a major proangiogenic player in both pathological and physiological angiogenesis $(15,27-29)$.

In brain glial tumors, grade-dependent VEGFR3 expression has been determined, meaning that it is absent in normal brain with scarce expression in low-grade astrocytoma (grade II) and greater expression in grade III and IV $(15,30,31)$.

The authors also observed rather the same findings.Venous Endothelial Growth Factor Receptor VEGF was expressed in all glioma grades with stronger intensity in high-grade tumors $(\mathrm{p}=0.052)$.
Targeting VEGF with Food and Drug Administration (FDA) approved mAb Avastin (bevacizamab) is one of the new classes of therapeutic agents via prevention of neovascularization of the tumor leading to a decrease in tumor size $(1,32)$.

Previous studies found that treatment of GBM with bevacizumab causes considerable reduction in tumor stem cells in a mouse model (9).

Investigations revealed that despite reduction in tumor size via VEGF blockade, there is no dramatic impact in tumor invasiveness $(1,33)$ and the outcome remains poor (6).

It has been suggested that VEGF inhibitors may be potentially combined with anti-PSMA antibodies (2, 34-38).

This can be explained because PSMA appears to induce VEGF independent angiogenesis $(2,39)$.

Expression of PSMA in GBM and more important in at least some lower grade gliomas, as observed in the current study, may provide a basis for more investigations focusing on selective treatment regimens in combination with the current standard care in all glioma grades, to improve treatment efficacy and tumor progression.

\section{Acknowledgment}

The authors would like to thank the staff of the pathology department of Shariati hospital for their devoted technical support.

\section{Conflict of Interest}

The authors declare no financial relationship with any organization regarding this research.

\section{References}

1. Carlsson SK, Brothers SP, Wahlestedt C. Enmerging treatment strategies for glioblastoma multiforme. EMBO Molecular Medicine. 2014;6(11):1359-70.

2. Nomura N, Pastorino S, Jiang P, et al. Prostate specific membrane antigen (PSMA) expression in primary gliomas and breast cancer brain metastasis. Caner Cell International. 2014;14:26.

3. Patel MA, Kim JE, Ruzevick J,et al. The Future of Glioblastoma Therapy: Synergism of Standard of Care and Immunotherapy Cancers. 2014;6:1953-85. 
4. DeAngelis L. Brain tumors. N England J Med. 2001;344:114-23.

5. Parsa A, Waldron J, Panner A, et al. Loss of tumor suppressor PTEN function increases B7-H1 expression and immunoresistance in glioma. Nat Med. 2006;13:84-8.

6. Wernicke AG, Edgar MA, Lavi E, et al. Prostate Specific Membrane Antigen as a potential Novel Vascular Target for Treatment of Glioblastoma Multiforme. Aarchives of Pathology and Laboratory Medicine. 2011;135:1486-9.

7. Shi W, Siemann D. Targeting the tumor vasculature: enhancing antitumor efficacy through combination treatment with ZD6126 and ZD6474. In Vivo. 2005;19(6):1045-50.

8. Burkhardt J, Hofstetter C, Santillan A, et al. Orthotopic glioblastoma stem-like cell xenograft model i mice to evaluateintra-arterial delivery of bevacizumab:from bedside to bench. J Clin Neurosci. 2012;19:1568-72.

9. Chen K, Huang Y-h, Chen J-1. Understanding and targeting cancer stem cells: therapeutic implications and challenges. Acta Pharmacologica Sinica. 2013;34:732-40.

10. pasqualini R, Arap W, McDonald D. Probing the stractural and molecular diversity $\mathrm{f}$ tumor vasculature. Trends Molecular Medicine. 2002;8(12):563-71.

11.Plate K, Risau W. Angigenesis in malignant gliomas. Glia. 1995;15(3):339-47.

12. Scappaticci F. Mechanisms and future directions for angiogenesis based cancer therapies. Journal of Clinical Oncology. 2002;20(18):390627.

13. Chang S, Reuter V, Heston W, Gaudin P. Metastatic Renal Cell Carcinoma neovasulature express Prostatic-Specific membrane antigen. Urology. 2001;57(4):801-5.

14. Gopalakrishnapillai A, Sonali PB, Jason $\mathrm{JC}$, et al. Assossiation of Prostate-specific membrane antigen with caveolin-1 and its caveoladependent internalization in microvascular endothelial cells:implications for targeting to tumor vasculture Microvascular research. 2006;72:45-61.
15. Grau SJ, Trillsch F, Luttichau Iv, et al. Lymphatic phenotype in tumor vessels in malignant gliomas. Neuropathology and Applied Neurobiology. 2008;34:675-9.

16. Bander N, Milowsky M, Nanus D, et al. Phase I trial of 177 lutetium-labeled J591, a monoclonal antibody to prostate-membrane antigen , in patients with androgen independent prostate cancer. Journal of Clinical Oncology. 2005;23(13):4591-601.

17.Liu H, Moy P, Kim S, al e. Monoclonal antibodies to the extracellular domain of prostatespecific membrane antigen also react with tumor vascular endothelium Cancer Research. 1997;57(17):3629-34.

18. Mhawech-Fauceglia $\quad \mathrm{P}, \quad$ Zhang $\mathrm{S}$, Terraccino L,et al. prostate-specific membrane antigen (PSMA) protein expression in normal and neoplastic tissues and its sensitivity and specificity in prostate adenocarcinoma: an immunohistochemical study using multiple tumor tissue micoarray technique Histopathology. 2007;50(4):472-83.

19. chang SS, Reuter VE, Heston WDW,et al. Five different Anti-Prostate- Specific Membrane Antigen (PSMA) antibodies confirm PSMA expression in tumor associated neovasculature. Cancer Research. 1999;59:3192.

20. Akhtar NH, Pail O, Saran A,et al. Prostate-Spaecific Membrane Antigen-Based Therapeutics. Advances in Urology. 2012;Article ID973820(doi:10.1155/2012/973820).

21. Troyler J, Beckett M, Jr GW. Detection and characterization of the prostate-specific membrane antigen(PSMA) in tissue extracts and body fluid,". International journal of Cancer. 1995;26(5):552-8.

22. Tsui $P$, Rubenstein N, Guinan $P$. Correlation between PSMA and VEGF expression as markers for LNCap tumor angiogenesis. Journal of Biomedicine and Biotechnology. 2005;3:287-90.

23. Milowsky M, Nanus D, Kostakoglu L,et al. Vn advanced solid tumorsascular targeted therapy with anti Prostate-specific membrane 
antigen monoclonal antibody J591 i. Journal of Clinical Oncology. 2007;25(5):540-7.

24. Morris M, Pandit-Taskar N, Divgi C,et al. Phase I evaluation of J591 as a vascular targeting agent in progressive solid tumors. Clinical Cancer Research. 2007;13(9):2707-13.

25.Haffner M, Kronberger I, Ross J, et al. Prostate-Specific Membrane Antigen expression in the neovasulature of gastric and colorectal cancers. Human Pathology. 2009;40:1754-62.

26. Sacha P, Zamecnik J, Barinka C, et al. Expression of glutamate carboxypeptidase II in human brain. NeuroScience. 2007;144:1361-72.

27. Kirsch M, Schackert G, Black P. Antiangiogenic treatment strategies for malignant brain tumours. Journal of Neurooncology. 2000;50:14963.

28. Roskoski R. Sanitinib: a VEGF and PDGF receptor protein kinase and angiogenesis inhibitor. Biochem Biophys Res Commun. 2007;356:323-8.

29. Takano S, Kamiyama H, Tsuboi K, Matsumura A. Angiogenesis and angiotherapy for malignant gliomas. Brain Tumor Pathology. 2004;21:69-73.

30. Grau S, Trillsch F, Herms J, et al. Expression of VEGFR3in glioma endothelium correlates with tumour grade. Journal of Neurooncology. 2007;82:141-50.

31.Jenny B, Harrison J, Baetens D, et al. Expression and localization of VEGF-C and VEGFR-3 in glioblastomas and haemangioblastomas. Journal of pthology. 2006;209:34-43.
32. Ferrara N, Hillan K, Novotny W. Bevacizumab(Avastin), a humanized anti-VEGF monoclonal antibody for cancer therapy. Biochem Biophys Res Commun. 2005;333:328-35.

33. Groot Jd, Fuller G, Kumar A, Piao Y, Eterovic K, Ji Y, et al. Tumor invasion after treatment of glioblastoma with bevacizumab:radiographic and pathologic correlation in humans and mice. Neuro-Oncol. 2010;12:233-42.

34. Brastianos $\mathrm{P}$, Bachelor T. VEGF inhibitors in brain tumors. Clin Adv Hematol Oncol. 2009; 7:753-60.

35. Carbonell W, Ansorge O, Sibson N, Muschel R. The vascular basement membrane as "soil" in brain metastasis. Plos One. 2009;4:e5857.

36. Jain R, diTomaso E, Duda D,et al. Angiogenesis in brain tumors. Nat Rev Neurosci. 2007;8:610-22.

37.Leenders W, Kusters B, Verrijp K, et al. Antiangiogenic therapy of cerebral melanoma metstases results in sustained tumor progression via vessel co-option. Clinical Cancer Research. 2004;10:6222-30.

38. Paez-Ribes M, Allen E, Hudock J, Takeda $\mathrm{T}$, et al. Antiangiogenic therapy elicits malignant progression of tumours to increased local invasion and distant metastasis. Cancer Cell. 2009;15:220-31.

39. Grant C, Caromile L, Ho V,et al. Prostate specific membrane antigen(PSMA) regulates angiogenesis independently of VEGF during ocular veovascularization. Plos One. 2012;7:e41285.

\section{How to Cite This Article}

Saffar, H., Noohi, M., Tavangar, S., Saffar, H., Azimi, S. Expression of Prostate-Specific Membrane Antigen (PSMA) in Brain Glioma and its Correlation with Tumor Grade. Iran J Pathol. 2018;13(1):45-53 